\title{
Existence of Follicular Helper T Cells in an Isolated IgG4-Related Hepatic Inflammatory Pseudotumor
}

\author{
Jack Song Chia ${ }^{1,2}$, Hung-Yen Chen ${ }^{1,3}$, Cheng-Maw Ho ${ }^{1,3}$, Yung-Ming Jeng ${ }^{4}$, Hui-Ling Chen ${ }^{3}$
}

1) Department of Surgery, National Taiwan University Hospital and College of Medicine, Taipei, Taiwan; 2) Royal College of Surgeons in Ireland (RCSI), Dublin, Ireland; 3) Hepatitis Research Center, National Taiwan University Hospital, Taipei, Taiwan; 4) Department of Pathology, National Taiwan University Hospital and College of Medicine, Taipei, Taiwan
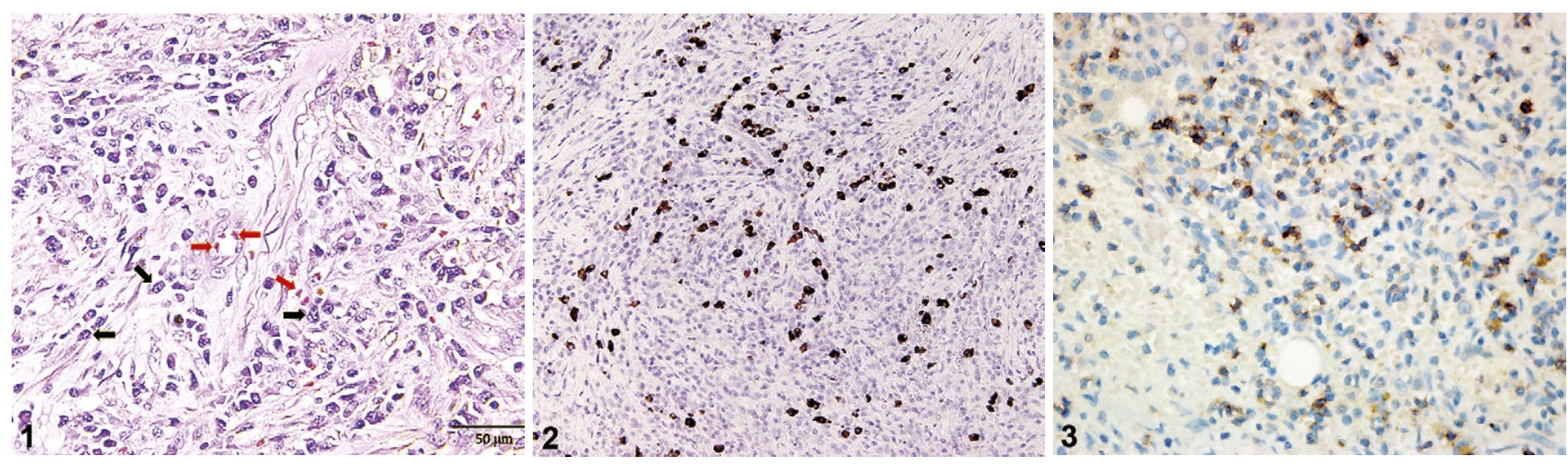

Hepatic inflammatory pseudotumor (HIP) is a benign mass comprised of infiltrating inflammatory/immune cells mimicking a malignant tumor. Hepatic inflammatory pseudotumor could be associated with systemic IgG4-related disease [1]. We present a patient with isolated IgG4-related HIP and showing the existence of follicular helper T cells in the tumor microenviroment.

A 53-years old man with a history of non-alcoholic fatty liver disease and diabetes mellitus was admitted to hospital due to multiple episodes of right flank pain and $6 \mathrm{~kg}$ weight loss over the past fortnight. A $2 \mathrm{~cm}$ hepatic tumor with typical enhancement pattern of hepatocellular carcinoma was resected.

The tumor was yellowish and elastic (Supplementary Fig S1). Histopathological examination excluded malignancy but showed the hazardous whorling pattern of fibroblasts (Supplementary Fig S2), eosinophils (red arrows), and plasma cells (black arrows) (Fig .1), which suggested the diagnosis of HIP. Isolated IgG4-related HIP was confirmed by a high proportion ( $>40 \%$ ) of IgG4 over all IgG expressing cells in immunohistochemical staining (Fig. 2, Supplementary Fig S3) and no involvement of extrahepatic organs. Moreover, follicular helper $\mathrm{T}$ (Tfh) cells expressed a large amount of programmed cell death-1 (PD-1) molecule (Fig. 3). Other cells involved in the microenviroment are described in the supplementary material.

Follicular helper $\mathrm{T}$ cells, recognized as an independent CD4+ $\mathrm{T}$ helper subset, specialized in helping $\mathrm{B}$ cells are crucial for affinity maturation and maintenance of humoral memory [2]. Highly expressing PD-1, Tfh cells are suggested to be critical in the pathogenesis of IgG4-related disease, particularly IgG4 class-switching, plasmablast and plasma cell differentiation, and germinal center formation $[3,4]$. Numerous lesional Tfh cells expressing PD-1 were, for the first time, identified in our case of isolated IgG4-related HIP, which was in accordance with the aforementioned hypothesis. We also demonstrated that lesional IgG4 accumulation at HIP can occur in the absence of systemic IgG4-related disease.

Corresponding author: Cheng-Maw Ho, miningho@ntu.edu.tw

Conflicts of interest: None to declare.

Acknowledgement: We thank Ms. Juo-Hsiang Pai and Dr. Ya-Hui Chen for their technical help.

\section{REFERENCES}

1. Stone JH, Zen Y, Deshpande V. IgG4-related disease. N Engl J Med 2012;366:539-551. doi:10.1056/NEJMra1104650

2. Vinuesa CG, Linterman MA, Yu D, MacLennan IC. Follicular helper T cells. Annu Rev Immunol 2016;34:335-368. doi:10.1146/annurevimmunol-041015-055605

3. Kamekura R, Takano K, Yamamoto M, et al. Cutting edge: A critical role of lesional $\mathrm{T}$ follicular helper cells in the pathogenesis of IgG4-related disease. J Immunol 2017;199:2624-2629. doi:10.4049/ jimmunol.1601507

4. Akiyama M, Suzuki K, Yasuoka H, Kaneko Y, Yamaoka K, Takeuchi T. Follicular helper T cells in the pathogenesis of IgG4-related disease. Rheumatology (Oxford) 2018;57:236-245. doi:10.1093/rheumatology/ kex171 